\title{
The Role of Calretinin, Inhibin, Melan-A, BCL-2, and C- kit in Differentiating Adrenal Cortical and Medullary Tumors: An Immunohistochemical Study
}

Paul J. Zhang, M.D., Elizabeth M. Genega, M.D., John E. Tomaszewski, M.D., Teresa L. Pasha, B.S., Virginia A. LiVolsi, M.D.

Department of Pathology and Laboratory Medicine, University of Pennsylvania Medical Center, Philadelphia, Pennsylvania

Morphologic distinction between adrenal cortical and medullary tumors can be difficult. Previous studies have shown inhibin, melan-A, and BCL-2 to be useful markers for adrenal cortical tumors. We have recently observed a high level of calretinin expression in normal adrenal cortex but not the medulla and therefore evaluated its diagnostic application for adrenal tumors in comparison with inhibin, melan-A, and BCL-2. C-kit is a transmembrane tyrosine kinase receptor. Immunodetection of c-kit expression has been recently used for tumor diagnosis, and c-kit-positive tumors can potentially benefit from kit kinase inhibitor treatment. Although c-kit expression was reported in adrenal medulla and pheochromocytoma, it has not been evaluated in adrenal cortical tumors. In this study, 28 adrenal cortical tumors (12 carcinomas, 16 adenomas), 20 pheochromocytomas, and 20 extraadrenal paragangliomas were evaluated for calretinin, inhibin, melan-A, BCL-2, and c-kit expression by standard immunohistochemical assays on paraffin sections. The percentage of immunoreactivity in adrenal cortical tumors was as follows: calretinin, 96\%; melan-A, 89\%; inhibin, 92\%; BCL-2, 20\%; and c-kit, 5\%. Normal adrenal medulla did not stain for c-kit but was positive for BCL-2. Eighty-six percent of pheochromocytomas stained for BCL-2 and none for calretinin, with the exception of the ganglioneuromatous areas in composite pheochromocytomas $(n=5)$. Extraadrenal paragangliomas showed reactivity with calretinin in $25 \%$, melan- $A$ in $5 \%$, inhibin in $16 \%, B C L-2$ in $38 \%$, and c-kit in $8 \%$ of the cases.

Copyright $\odot 2003$ by The United States and Canadian Academy of Pathology, Inc.

VOL. 16, NO. 6, P. 591, 2003 Printed in the U.S.A.

Date of acceptance: March 28, 2003.

Address reprint requests to: Paul J. Zhang, M.D., Anatomic Pathology, 6 Founders Pavilion, University of Pennsylvania Medical Center, 3400 Spruce Street, Philadelphia, PA 19147; fax: 215-349-5910; e-mail: pjz@mail.med.upenn.edu.

DOI: 10.1097/01.MP.0000073134.60541.E8
Our results indicate that calretinin is the most sensitive among all the adrenal markers tested. Like melan-A and inhibin, calretinin is also a very specific marker in differentiating cortical from medullary adrenal tumors. In addition, calretinin can be used to confirm a composite pheochromocytoma. BCL-2 does not appear to be useful in differentiating adrenal cortical from medullary tumors. C-kit is not useful in the diagnosis of adrenal tumors, and kit kinase inhibitor might have a limited role in the treatment of adrenal tumors and paraganglioma because of the low frequency of c-kit expression in these tumors.

KEY WORDS: Adrenocortical tumors, BCL-2, Calretinin, C-kit, Paraganglioma, Pheochromocytoma. Mod Pathol 2003;16(6):591-597

Morphologic distinction between adrenal cortical and medullary lesions can be difficult. Previous studies have shown inhibin $\alpha$-subunit, melan-A, BCL-2, and lately calretinin to be useful as markers of adrenal cortical differentiation in tumor diagnosis (1-6). In the current study, we accessed the value of these markers and examined the immunoreactivity for c-kit in tumors of adrenal origin to expand the knowledge of a useful panel of adrenal cortical markers. The selection and application of an immunohistochemical panel in the diagnosis of adrenocortical tumors is discussed.

Calretinin, a calcium-binding protein, was first discovered in neural tissue, later in mesothelium and ovarian sex-cord stromal tissue; it has been used as a marker for tumors of these origins (7-11). High levels of calretinin have been observed recently in normal adrenal cortex but not in the medulla (unpublished observation). Recently, calretinin has been shown to be positive in $73 \%$ of adrenal cortical tumors (6). In this study, we intended to determine whether calretinin was a more superior discriminator of adrenal cortical from medullary 


\begin{tabular}{llll}
\hline \multicolumn{1}{c}{ Antibody } & \multicolumn{1}{c}{ Source } & \multicolumn{1}{c}{ Clone \& Titer } & \multicolumn{1}{c}{ Antigen Retrieval } \\
\hline Calretinin & Zymed, So. San Francisco, CA & Polyclonal, 1:25 & Microwave, 8 minutes, IX citrate buffer \\
Inhibin-alpha & Oxford Bio-Innovation, UK & R1, 1:25 & Microwave, 8 minutes, IX citrate buffer \\
Melan-A & Dako, Carpinteria, CA & A130, 1:25 & Microwave, 8 minutes, target retrieval solution \\
c-kit & Dako & Polyclonal, 1:100 & Microwave, 8 minutes, IX citrate buffer \\
BCL-2 & Dako & $124,1: 50$ & Steamer, 40 minutes \\
\hline
\end{tabular}

tumors than the previously defined adrenal markers such as inhibin, melan-A, and BCL-2.

C-kit (CD117), a transmembrane tyrosine kinase receptor, plays an important role in tyrosine phosphorylation of protein substrates, with subsequent activation of intracellular signaling cascades controlling cell proliferation, apoptosis, and differentiation (12). Expression of c-kit is seen in a variety of normal human tissues, such as mast cells and intestinal Cajal cells and their corresponding neoplasms (13-15). Recently, kit kinase inhibitors have been shown to have promising therapeutic effect on c-kit-expressing tumors (16). Immunodetection of c-kit expression has become important in the diagnosis and treatment of these c-kit-expressing tumors. Previous studies have shown c-kit expression in adrenal medulla in animal and human tissue $(13,15)$. Using an immunohistochemical assay on frozen tissue, Matsuda et al. (15) found c-kit immunoreactivity in the nuclei of the adrenal medulla and pheochromocytoma. However, c-kit has not been evaluated in adrenal cortical and medullary tumors in formalin-fixed, paraffinembedded tissue. We evaluated the immunoreactivity of calretinin, c-kit, inhibin, melan-A, and BCL-2 in a series of adrenal tumors and paraganglioma in routine surgical specimens.

\section{MATERIALS AND METHODS}

Paraffin blocks of 28 adrenal cortical tumors (12 carcinomas, 16 adenomas), 20 pheochromocytomas, and 20 extraadrenal paragangliomas were retrieved from the surgical pathology files at Hospital of the University of Pennsylvania. Immunohistochemical stains were performed on paraffin sections using antibodies against calretinin (poly-

Table 2. Results of Immunohistochemical Evaluation

\begin{tabular}{|c|c|c|c|c|c|}
\hline Diagnosis & Calretinin & Melan-A & Inhibin & BCL-2 & c-kit \\
\hline Cortical carcinoma & $N=12$ & $N=12$ & $N=12$ & $N=10$ & $N=9$ \\
\hline Negative & $1(8 \%)$ & $3(25 \%)$ & $1(8 \%)$ & $7(70 \%)$ & $7(89 \%)$ \\
\hline Positive (total) & $11(92 \%)$ & $9(75 \%)$ & $11(92 \%)$ & $3(30 \%)$ & $1(11 \%)$ \\
\hline $\mathrm{F}+$ & $2(17 \%)$ & $4(33 \%)$ & 0 & 0 & 0 \\
\hline $1+$ & $3(25 \%)$ & $3(25 \%)$ & $7(58 \%)$ & $2(20 \%)$ & 0 \\
\hline $2+$ & 0 & 0 & $2(17 \%)$ & $1(10 \%)$ & $1(11 \%)$ \\
\hline $3+$ & $6(50 \%)$ & $2(17 \%)$ & $2(17 \%)$ & 0 & 0 \\
\hline Cortical adenoma & $N=16$ & $N=15$ & $N=15$ & $N=15$ & $N=13$ \\
\hline Negative & 0 & 0 & $1(7 \%)$ & $13(93 \%)$ & $13(100 \%)$ \\
\hline Positive (total) & $16(100 \%)$ & $15(100 \%)$ & $14(93 \%)$ & $2(13 \%)$ & 0 \\
\hline $\mathrm{F}+$ & 0 & $2(13 \%)$ & $4(27 \%)$ & $1(7 \%)$ & 0 \\
\hline $1+$ & $3(19 \%)$ & $3(27 \%)$ & $8(53 \%)$ & 0 & 0 \\
\hline $2+$ & $5(31 \%)$ & $6(40 \%)$ & $2(13 \%)$ & $1(7 \%)$ & 0 \\
\hline $3+$ & $8(50 \%)$ & $3(20 \%)$ & 0 & 0 & 0 \\
\hline Pheochromocytoma & $N=20$ & $N=18$ & $N=18$ & $N=14$ & $N=14$ \\
\hline Negative & $20 *(100 \%)$ & $16(89 \%)$ & $18(100 \%)$ & $2(14 \%)$ & $12(86 \%)$ \\
\hline Positive (total) & 0 & $2(11 \%)$ & 0 & $12(86 \%)$ & $2(14 \%)$ \\
\hline $\mathrm{F}+$ & 0 & $1(6 \%)$ & 0 & 0 & $2(14 \%)$ \\
\hline $1+$ & 0 & $1(6 \%)$ & 0 & $6(43 \%)$ & 0 \\
\hline $2+$ & 0 & 0 & 0 & $4(29 \%)$ & \\
\hline $3+$ & 0 & 0 & 0 & $2(14 \%)$ & 0 \\
\hline Paraganglioma & $N=20$ & $N=19$ & $N=19$ & $N=13$ & $N=12$ \\
\hline Negative & $15(75 \%)$ & $18(95 \%)$ & $16(84 \%)$ & $8(62 \%)$ & $11(92 \%)$ \\
\hline Positive (total) & $5(25 \%)$ & $1(5 \%)$ & $3(16 \%)$ & $5(38 \%)$ & $1(8 \%)$ \\
\hline $\mathrm{F}+$ & $2(10 \%)$ & 0 & $1(5 \%)$ & 0 & 0 \\
\hline $1+$ & 0 & $1(5 \%)$ & $2(11 \%)$ & $2(15 \%)$ & $1(8 \%)$ \\
\hline $2+$ & $3(15 \%)$ & 0 & 0 & $3(23 \%)$ & 0 \\
\hline $3+$ & 0 & 0 & 0 & 0 & 0 \\
\hline
\end{tabular}

$\mathrm{F}+:<10 \%$, weak or strong intensity.

$1+:>$ or $=10 \%<25 \%$, weak or strong intensity.

$2+:>$ or $=25 \%$ and $<50 \%$, weak or strong intensity; or $>$ or $=50 \%$, weak intensity.

$3+:>50 \%$, strong intensity. 
clonal, 1:25; Zymed, So. San Francisco, CA), inhibin $\alpha$-subunit (clone R1, 1:25; Oxford Bio-Innovation, Oxfordshire, UK), melan-A (clone A103, 1:25; DAKO, Carpenteria, CA), BCL-2 (clone 124, 1:50; DAKO), and c-kit (polyclonal, 1:100, DAKO). Immunostains for calretinin, melan-A, and inhibin were performed with avidin-biotin peroxidase complex method on a Biotek autostainer (Techmate 1000; Ventana, Tucson, AZ), and c-kit and BCL-2 were performed with an Envision kit (DAKO) on a DAKO autostainer. Sections were pretreated in a microwave oven $(1100 \mathrm{~W})$ at $70 \%$ power for 8 minutes in $1 \times$ citrate buffer at $\mathrm{pH} 6.0$ (Lab Vision, Fremont, CA) for anti-calretinin, inhibin, and c-kit and in $1 \times$ target retrieval solution (DAKO) for melan-A. Sections for BCL-2 immunostaining were steamed in $1 \times$ citrate buffer at $\mathrm{pH} 6.0$ (Lab Vision, Fremont, $\mathrm{CA})$ at $95^{\circ} \mathrm{C}$ for 40 minutes in a Black and Decker Steamer. Antibody information is summarized in Table 1. Tissue of mesothelioma, normal testis, melanoma, tonsil, and gastrointestinal stromal tumor were used for positive controls for calretinin, inhibin, melan-A, BCL-2, and c-kit, respectively. Primary antibody was substituted by normal mouse or rabbit serum in a section of each case as a negative control.

Immunoreactivity was evaluated semiquantatively under light microscope and scored as negative, $\mathrm{F}+(<10 \%$, weak or strong intensity), $1+$ ( $\geq 10 \%$ and $<25 \%$, weak or strong intensity), $2+$ ( $\geq 25 \%$ and $<50 \%$, weak or strong intensity; or $\geq 50 \%$, weak intensity) and $3+(>50 \%$, strong intensity) accordingly.

\section{RESULTS}

Adrenal cortical tumors showed cytoplasmic immunoreactivity in $96 \%$ of the cases for anticalretinin, $89 \%$ for anti-melan-A and $92 \%$ for antiinhibin, $20 \%$ for anti-BCL-2, and $5 \%$ for anti-c-kit. Immunoreactivity with anti-calretinin, antimelan-A, and anti-inhibin was identified in $92 \%$, $75 \%$, and $92 \%$ of adrenocortical carcinomas and $100 \%, 100 \%$, and $93 \%$ of adenomas, respectively (Table 2). Among the three adrenal cortical markers tested, calretinin immunoreactivity tended to be the most sensitive and more intense and diffuse in staining pattern than melan-A and inhibin (Fig. 1).
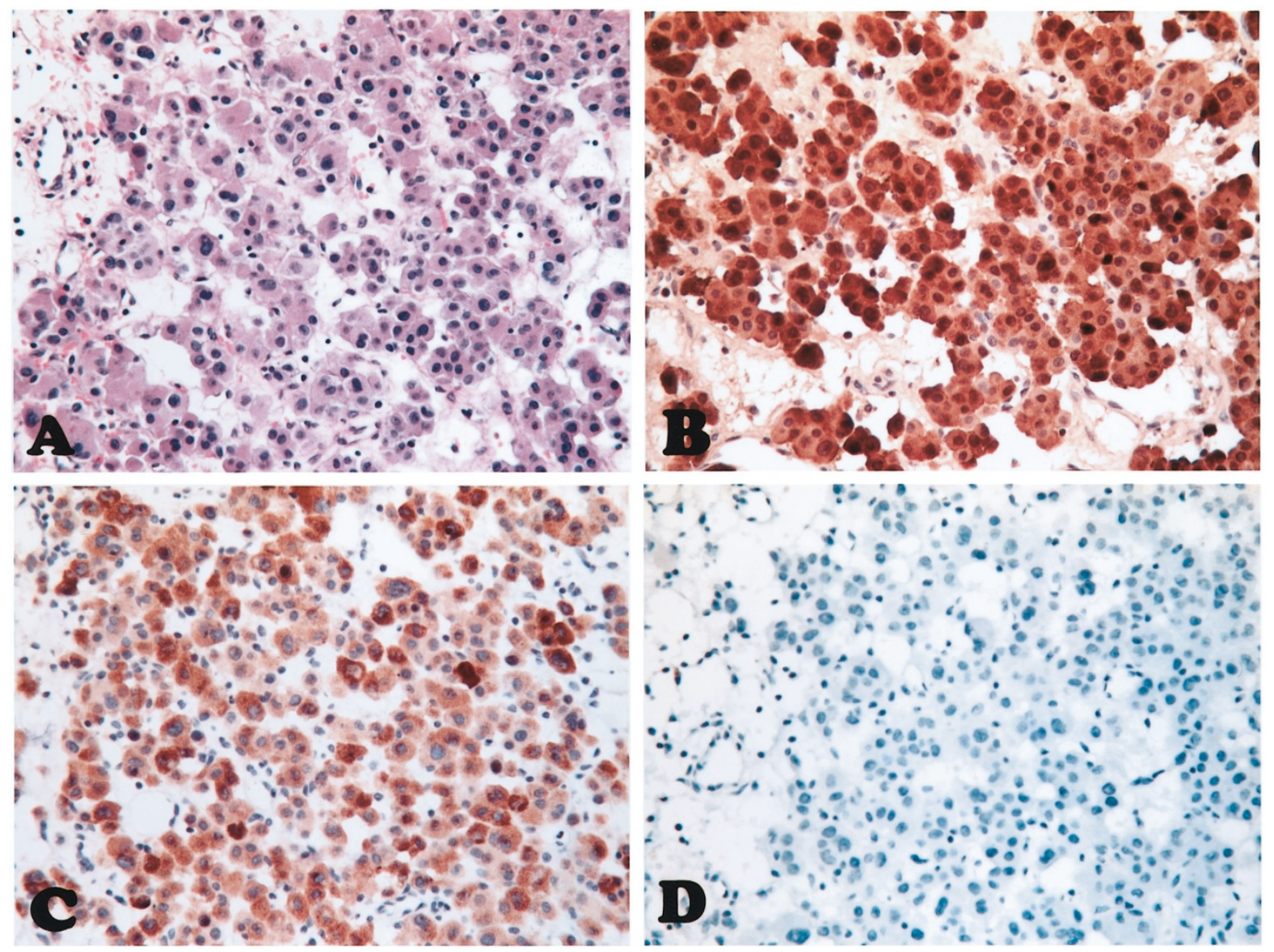

FIGURE 1. An adrenocortical carcinoma (A) showing immunoreactivity with anit-calretinin (B), melan-A (C), and inhibin (D), with calretinin immunoreactivity being the strongest and inhibin being negative. Note cytoplasmic and nuclear staining in calretinin (B) and melan-A (C) immunostaining. 


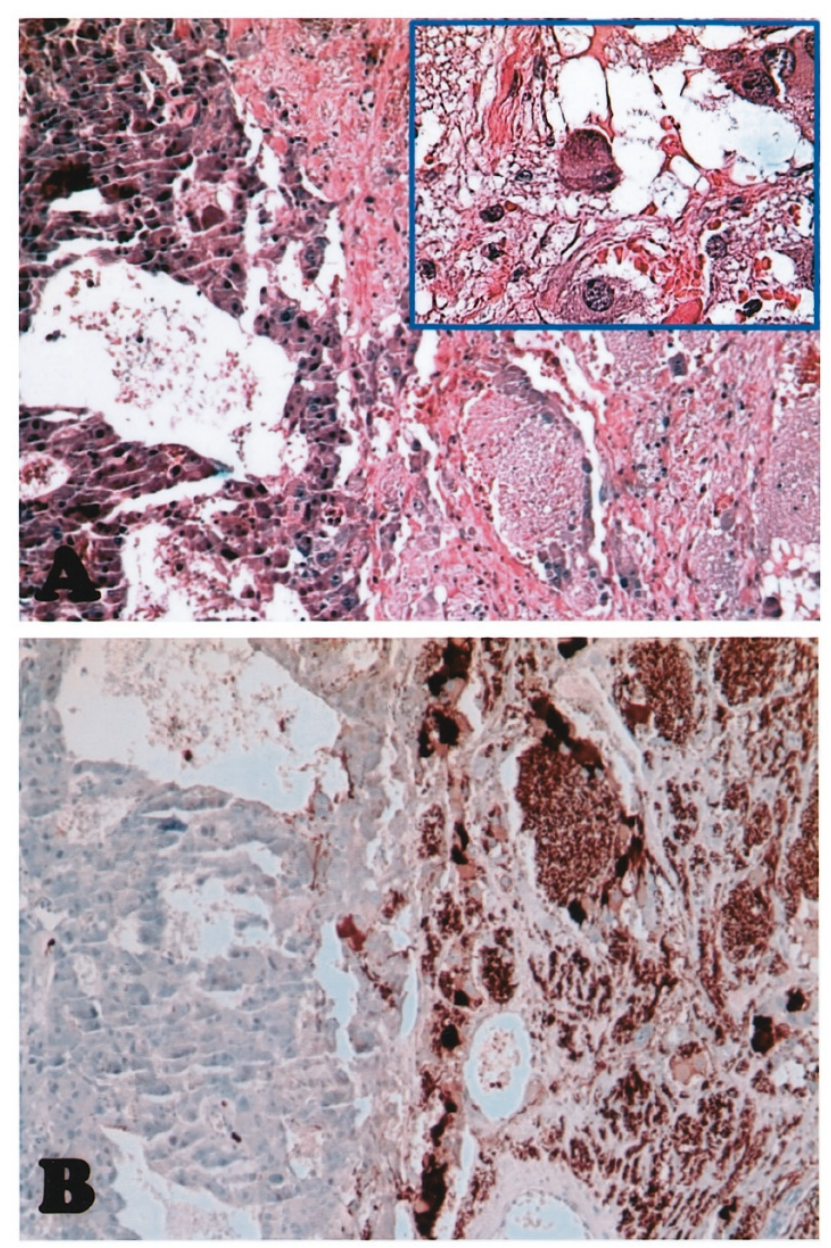

FIGURE 2. A, composite pheochromocytoma. B, calretinin immunoreactivity in the ganglioneuromatous area of the composite pheochromocytoma but not in the pheochromocytes in the left half field.

In addition to cytoplasmic staining, nuclear immunostaining was also seen frequently with calretinin and less frequently with melan-A immunostaining (Fig. 1). Although carcinomas were slightly less immunoreactive than adenomas, no single adrenal cortical tumor was negative for all three markers or positive for only one marker. BCL-2 cytoplasmic immunoreactivity was detected only in three cases (30\%) of adrenocortical carcinomas and 2 of 15 (13\%) adrenocortical adenomas. C-kit immunoreactivity was detected in 1 of $9(11 \%)$ adrenocortical carcinomas and 0 of 13 adrenocortical adenomas.

Pheochromocytomas were generally negative for calretinin, with the exception of the ganglioneuroma areas in composite pheochromocytomas (Fig. 2 ), and were negative for inhibin. Focal to $1+$ melan-A reactivity was present in two pheochromocytomas $(11 \%)$. BCL-2 cytoplasmic immunoreactivity was detected in $12 / 14(86 \%)$ pheochromocytomas (Fig. 3). Focal c-kit immunoreactivity was detected in $2(14 \%)$ of 14 pheochromocytomas. In extraadrenal paragangliomas, 5 of 20 cases $(25 \%)$ were positive for calretinin $(\mathrm{F}+$ to $2+), 1$ of $19(5 \%)$ for melan-A (1+), 3 of $19(16 \%)$ for inhibin ( $\mathrm{F}+$ to $1+), 5$ of $13(38 \%)$ for BCL-2 (1+ and $2+)$, and 1 of $12(8 \%)$ for c-kit $(1+)$ (Table 1). All layers of the normal adrenal cortex, when present adjacent to the adrenal tumors, were variably positive for calretinin, melan-A, and inhibin without specific zonal staining pattern (Fig. 4). BCL-2 immunoreactivity was present primarily in normal adrenal medulla and lymphocytes adjacent to or entrapped in the adrenal tumors (Fig. 3). In contrast to the strong reactivity in the medulla, weak and rare granular cytoplasmic BCL-2 immunoreactivity was occasionally seen in the cortex. No c-kit immunoreactivity was detected in any normal adrenal cortex and medulla.

\section{DISCUSSION}

We have shown strong and frequent calretinin immunoreactivity in normal adrenal cortex and benign and malignant adrenocortical tumors. Previously, $50-100 \%$ of adrenocortical carcinomas and $68-100 \%$ of adrenocortical adenomas were found to be positive for melan-A, $73-100 \%$ and $86-100 \%$ for inhibin, and 95\% for BCL-2 (1-5). Most recently, Jorda et al. (6) also reported calretinin immunoreactivity in $73 \%$ of adrenocortical tumors they studied. In comparison to these previous studies, we have shown that $96 \%, 89 \%, 92 \%$, and $20 \%$ of adrenocortical tumors were positive for calretinin, melan-A, inhibin, and BCL-2, respectively, with calretinin being the most sensitive of all in identifying adrenal cortical tumors. Although pheochromocytomas and paragangliomas are generally negative for calretinin, melan-A, and inhibin, we found calretinin immunoreactivity in the ganglioneuromatous areas of composite pheochromocytoma. Because of its known immunoreactivity in neural tissue, it is not surprising to find calretinin immunoreactivity in ganglioneuromatous areas of composite pheochromocytomas $(7,9)$. One should be aware of these calretinin-positive ganglioneuromatous areas in composite pheochromocytoma in order to avoid interpreting them as evidence of adrenocortical tissue or tumor. Instead, calretinin can be used to confirm the presence of ganglioneuromatous areas in a composite pheochromocytoma while differentiating cortical cells from medullary cells in adrenal tumors.

BCL-2 was shown to be frequently positive in adrenocortical tumors and negative in medullary tissue and pheochromocytomas in a previous study (5). In this study, BCL-2 immunoreactivity was validated by a mantle zone pattern of staining in tonsil tissue and the entrapped lymphocytes in tumor tissues as external and internal positive controls 




FIGURE 3. A: BCL2 immunoreactivity in the mantle zones of germinal centers in tonsilar tissue used as BCL 2 positive control. B: Strong cytoplasmic BCL-2 immunoreactivity in a pheochromocytoma but not in the adjacent normal cortex in the left lower field. C and D: Lack of BCL-2 immunoreactivity in an adrenal cortical adenoma with the entrapped BCL-2 positive lymphocytes. E and F: Inhibin (E) and BCL-2 (F) immunoreactivity reservedly highlights the adrenal cortex and medulla respectively in a normal adrenal gland.

(Figure 3). However, BCL-2 immunoreactivity was detected focally in only $20 \%$ of the adrenal cortical tumors but more diffusely in $86 \%$ of pheochromocytomas and in adrenal medulla. Although the same monoclonal antibody was used in both studies, we have diluted the antibody at 1:50 and used a steamer for antigen retrieval. In contrast, the titer of the primary antibody was more concentrated (1: 20 ), and the combined pressure cooker-microwave heating method for antigen retrieval was harsher on tissue in the study of Fogt et al. (5). The discrepancy in BCL-2 immunoreactivity in adrenal tissue and 


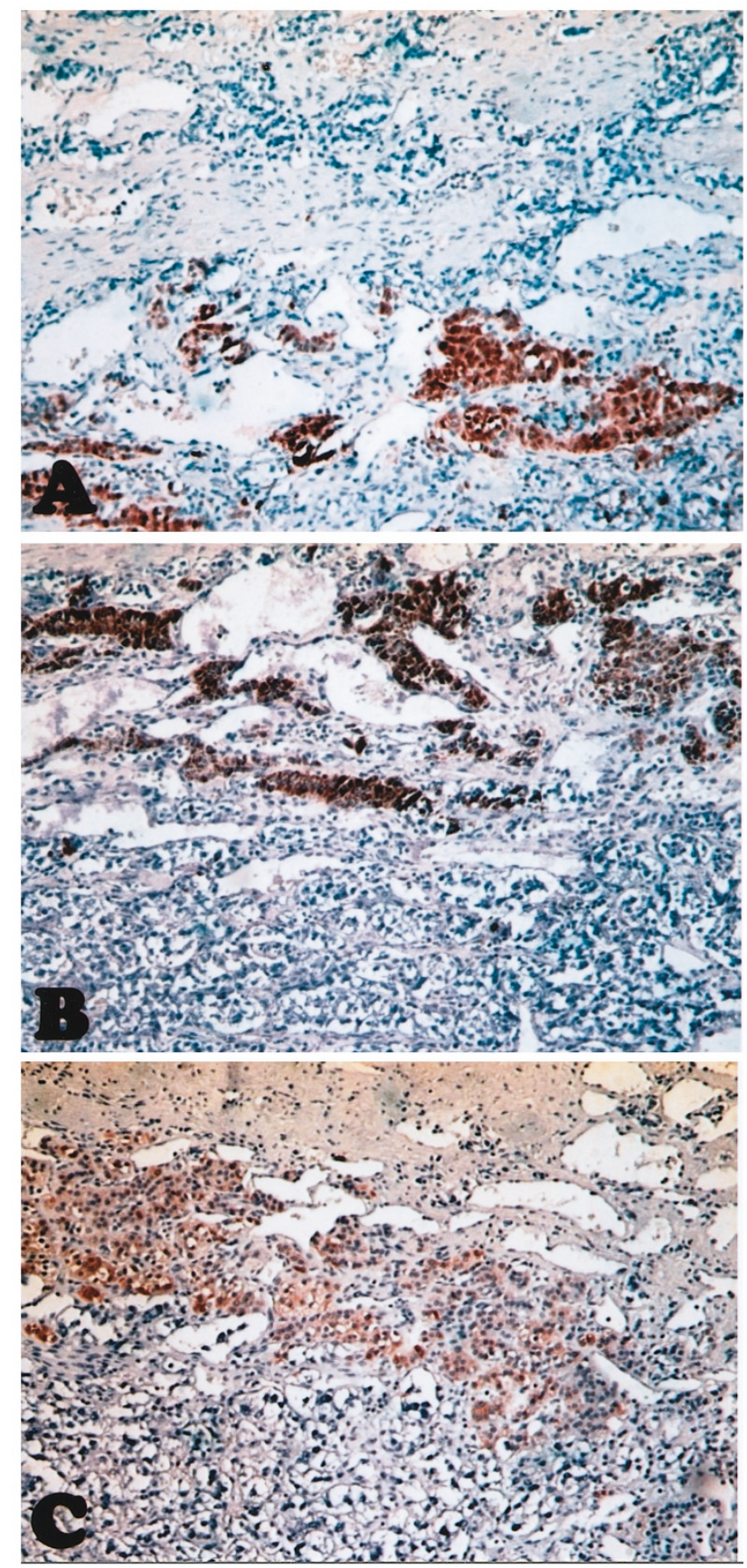

FIGURE 4. Immunoreactivity with anti-calretinin (A), melan-A (B), and inhibin $(\mathrm{C})$ in normal adrenal cortical tissue entrapped in a pheochromocytoma.

tumors is not clear, but this likely is due to the different heating methods used for antigen retrieval. Studies by other methodologies such as absorption, Western blotting, and molecular techniques are needed to further investigate BCL-2 expression in adrenal tissues and tumors.

Both pheochromocytoma and adrenocortical tumor can express certain neuroendocrine markers such as synaptophysin and neuron-specific enolase but not chromogranin, which is characteristically positive in pheochromocytoma but negative in ad- renocortical tumors $(17,18)$. Although pheochromocytoma can be differentiated from adrenocortical tumors by chromogranin positivity, in the differential diagnosis of an adrenocortical tumor versus medullary tumor, one can apply at least one of the adrenocortical markers to confirm the cortical origin of the tumor in addition to chromogranin, which can only be used to exclude but not confirm an adrenocortical origin. Although calretinin, melan-A, and inhibin are highly sensitive and specific in differentiating adrenal cortical tumors from medullary tumors, none are highly specific for adrenal cortical tumors. Melan-A and inhibin both are known to be positive in a number of nonadrenal tumors, such as melanomas and angiomyolipomas (melan-A) and ovarian epithelial and sex-cord stromal tumors (melan-A and inhibin; 19-25). Similarly, calretinin immunoreactivity is also seen in a few extraadrenal tumors such as mesotheliomas, ovarian sex-cord stromal tumors, and some neural tumors $(8,10,11,21)$. Different from adrenocortical tumors, the above extraadrenal tumors are also known to coexpress other markers such as $\mathrm{S} 100$ in melanoma and other neural tumors, HMB45 in melanoma and angiomyolipoma, actin in angiomyolipoma, cytokeratin in mesothelial or epithelial tumor, and CD99 in sex-cord stromal tumor of the gonads (8, $21,22,24,25)$. Both sex-cord stromal tissue and adrenal cortical tissue are steroidogenic (26). It is of great interest that tumors arising from the steroidogenic adrenal cortex and sex-cord stroma of the gonads express inhibin, melan-A, and calretinin. Different from adrenocortical tumors, however, ovarian sex-cord stromal tumors are also positive for CD99 that is not known in adrenal cortical tumors $(24,25)$.

The adrenal medulla belongs to the synthetic nerve system and is the site for catecholamine production. There have been few markers specific for the adrenal medulla and its tumors on paraffin section. Although c-kit expression was reported in normal adrenal medulla and pheochromocytoma in previous studies, we could not detect any c-kit immunoreactivity in normal adrenal medulla and pheochromocytomas on paraffin sections. No c-kit immunoreactivity was identified in any of the paragangliomas or adrenocortical tumors.

In summary, in addition to normal adrenal cortex, calretinin is also most frequently expressed in both benign and malignant adrenocortical tumors among all the adrenal markers tested. It is sensitive and specific in differentiating tumors of adrenocortical origin from those of adrenal medullary origin and is useful in the diagnosis of composite pheochromocytoma. In addition to being more frequent, calretinin staining tends to be more intensive and diffuse than do inhibin and melan-A in adrenocortical tumors. Calretinin appears to be superior to inhibin and melan-A in the diagnosis of adrenocortical tumor when tissue heterogeneity becomes an 
issue in small tissue sampling and a more costeffective panel is desirable. In addition to the above adrenal markers, the antibody panel should always include other relevant non-adrenocortical markers, depending on the scope of the differential diagnosis, to improve the specificity of the immunohistochemical workup. BCL-2 should not be used for differential diagnosis of an adrenal tumor on paraffin section until further studies are performed to clarify the site of its expression in adrenal tumors. C-kit is not a useful marker for adrenal medulla and its tumors. Because of the low frequency of c-kit expression, kit kinase inhibitors may have a limited therapeutic role in adrenal cortical and medullary tumors as well as in extraadrenal paraganglioma.

\section{REFERENCES}

1. Cho EY, Ahn GH. Immunoexpression of inhibin $\alpha$-subunit in adrenal neoplasms. Appl Immunohistochem Mol Morphol 2001;9:222-8.

2. Busam KJ, Iversen K, Coplan KA, Old LJ, Stockert E, Chen YT, et al. Immunoreactivity for A103, an antibody to melan-A (Mart-1), in adrenocortical and other steroid tumors. Am J Surg Pathol 1998;22:57-63.

3. Pelkey TJ, Frierson HF, Mills SE, Stoler MH. The alpha subunit of inhibin in adrenal cortical neoplasia. Mod Pathol 1998;11:516-24.

4. Renshaw AA, Granter SR. A comparison of A103 and inhibin reactivity in adrenal cortical tumors: distinction from hepatocellular carcinoma and renal tumors. Mod Pathol 1998;11: $1160-4$.

5. Fogt F, VortmeyerPoremba C, Minda M, Harris CA, Tomaszewski JE. Bcl-2 Expression in normal adrenal glands and in adrenal neoplasms. Mod Pathol 1998;11:716-20.

6. Jorda M, De Madeiros B, Nadji M. Calretinin and inhibin are useful in separating adrenocortical neoplasms from pheochromocytomas. Appl Immunohistochem Mol Morphol 2002;10:67-70

7. Papka RE, Collins J, Copelin T, Wilson K. Calretininimmunoreactive nerves in the uterus, pelvic autonomic ganglia, lumbrosacral dorsal root ganglia and lumbrosacral spinal. Cell Tissue Res 1999;298:63-74.

8. Doglioni C, Tos AP, Laurino L, Iuzzolino P, Chiarelli C, Celio MR, et al. Calretinin: a novel immunocytochemical marker for mesothelioma. Am J Surg Pathol 1996;20:1037-46.

9. Rogers JH. Calretinin: a gene for a novel calcium-binding protein expressed principally in neurons. J Cell Biol 1987; 105:1343-53.

10. Attanoos RL, Dojcinov SD, Webb R, Gibbs AR. Antimesothelial markers in sarcomatoid mesothelioma and other spindle cell neoplasms. Histopathology 2000;37:22432 .
11. McCluggage WG, Maxwell P. Immunohistochemical staining for calretinin is useful in the diagnosis of ovarian sex cordstromal tumors. Histopathology 2001;38:403-8.

12. Taylor ML, Metcalfe DD. Kit signal transduction. Hematol Oncol Clin North Am 2000;14:517-35.

13. Kim JS, Kubota H, Nam SY, Doi K, Saegusa J. Expression of cytokines and proteases in mast cells in the lesion of subcapsular cell hyperplasia in mouse adrenal glands. Toxicol Pathol 2000;28:297-303.

14. Sarloma-Rikala M, Kovatich AJ, Barusevicius A, Miettinen M. CD117: a sensitive marker for gastrointestinal stromal tumors that is more specific than CD34. Mod Pathol 1998;11: 723-34.

15. Matsuda R, Takahashi T, Nakamura S, Sekido Y, Nishida K, Seto M, et al. Expression of the c-kit protein in human solid tumors and in corresponding fetal and adult normal tissues. Am J Pathol 1993;142:339-46.

16. Wang WL, Healy ME, Sattler M, Verma S, Lin J, Maulik G, et al. Growth inhibition and modulation of kinase pathways of small cell lung cancer cell lines by the novel tyrosin kinase inhibitor STI 571. Oncology 2000;19:3521-8.

17. Komminoth P, Roth J, Schroder S, Saremaslani P, Heitz PU. Overlapping expression of immunohistochemical markers and synaptophysin mRNA in pheochromocytomas and adrenocortical carcinomas. Implications for the differential diagnosis of adrenal gland tumors. Lab Invest 1995;72:424-31.

18. Alsabeh R, Mazoujian G, Goates J, Medeiros LJ, Weiss LM. Adrenal cortical tumors clinically mimicking pheochromocytoma. Am J Clin Pathol 1995;104:382-90.

19. McCluggage WG, Maxwell P, Patterson A, Sloan JM. Immunohistochemical staining of hepatocellular carcinoma with monoclonal antibody against inhibin. Histopathology 1997; 30:518-22.

20. Yamashita K, Yamoto M, Shikone T, Minami S, Nakano R. Immunohistochemical localization of inhibin and activin subunits in human epithelial ovarian tumors. Am J Obstet Gynecol 1999;180:316-22.

21. Murakata LA, Ishak KG. Expression of Inhibin- $\alpha$ by granular cell tumors of the gallbladder and extrahepatic bile ducts. Am J Surg Pathol 2001;25:1200-3.

22. Jungbluth AA, Iversen K, Coplan K, Williamson B, Chen YT, Stockert E, et al. Expression of melanocyte-associated markers gp-100 and Melan-A/MART-1 in angiomyolipomas. An immunohistochemical and rt-PCR analysis. Virchows Arch 1999;434:429-35.

23. Stewart CJ, Nandini CL, Richmond JA. Value of A103 (melan-A) immunostaining in the differential diagnosis of ovarian sex cord stromal tumors. J Clin Pathol 2000;53:20611.

24. Choi YL, Kim HS, Ahn G. Immunoexpression of inhibin alpha subunit, inhibin/activin betaA subunit and CD99 in ovarian tumors. Arch Pathol Lab Med 2000;124:563-9.

25. Matias-Guiu X, Pons C, Prat J. Mullerian inhibiting substance, alpha-inhibin, and CD99 expression in sex cordstromal tumors and endometrioid ovarian carcinomas resembling sex cord-stromal tumors. Hum Pathol 1998;29: 840-5.

26. Morohashi K. The ontogenesis of the steroidogenic tissues. Genes Cells 1997;2:95-106. 